A) Check for updates

Cite this: Food Funct., 2021, 12, 2962

\title{
Wheat bran fermented by mixed fungal strains improves the digestibility of crude fiber and may benefit the gut health without impacting the growth performance in weaned pigs $\dagger$
}

\author{
Yuheng Luo, D $* \psi^{\mathrm{a}}$ Jun He, $\psi^{\mathrm{a}}$ Hua Li,,$^{\mathrm{a}}$ Cong Lan, ${ }^{\mathrm{a}}$ Jingyi Cai, ${ }^{\mathrm{a}}$ Hong Chen, ${ }^{\mathrm{b}}$ \\ Gang Tian, ${ }^{a}$ Huifen Wang, ${ }^{a}$ Quyuan Wang, ${ }^{a}$ Jun $\mathrm{He}$, (1) ${ }^{\mathrm{a}}$ Daiwen Chen, ${ }^{\mathrm{a}}$ Bing Yu, (10 ${ }^{a}$ \\ Zhiqing Huang, ${ }^{a}$ Ping Zheng, ${ }^{a}$ Xiangbing Mao, (D) a Jie Yu, ${ }^{a}$ Junqiu Luo, ${ }^{a}$ Aimin $\mathrm{Wu}^{a}$ \\ and Hui Yan ${ }^{a}$
}

\begin{abstract}
This study was conducted to compare the effect of raw (WB) or mixed fungi-fermented wheat bran (FWB) on the growth, nutrient digestibility and intestinal health in weaned piglets. After the preparation of FWB, twenty-one cross-bred weaned piglets $(7.20 \pm 0.5 \mathrm{~kg})$ were separated into three groups for a 40 -day trial. The pigs in the control group were fed a basal corn-soybean meal diet. For the other two groups, $8 \%$ of expanded corn in the basal diet was replaced by equivalent WB or FWB. Results showed that the content of main nutrients and the composition of dietary fiber in FWB improved compared to that for WB. The digestibility of fiber in pigs fed FWB improved $(P<0.05)$ compared to the control and/or WB without affecting their growth performance. Both WB and FWB decreased the conditional pathogen (Streptococcus) or/and E. coli virulence factor (STb) in the colon compared to control $(P<0.05)$, and the ratio of villus height to crypt depth (VCR) in jejunum increased $(P<0.05)$. The number of goblet cells, the expression of MUC-1 and PBD1 in jejunal mucosa, and the proportion of blood CD4 $4^{+}$T lymphocyte subset improved $(P<0.05)$ by FWB rather than WB. Furthermore, although only WB elevated $(P<0.05)$ the concentration of butyrate in the colon, both WB and FWB increased the number of butyrate-producing bacteria $(P<0.05)$ compared to the control. Thus, the main advantage of FWB over WB in weaned pigs is its improvement in fiber digestibility.
\end{abstract}

Received 27th January 2021, Accepted 22nd February 2021 DOI: $10.1039 / \mathrm{d} 1 \mathrm{fo} 00273 \mathrm{~b}$

rsc.li/food-function
NSP contains a large number of beta-1,4 glycosidic bonds, which cannot be directly utilized by mono-gastric animals due to the lack of carbohydrate active enzymes (CAZys). ${ }^{3}$ Conversely, nutrients can be easily wrapped by the high-viscosity NSP, which blocks the enzymatic reaction and leads to a decrease in digestibility. ${ }^{4}$ Thus, the high content of NSP in WB limits its use in the feed of piglets, which often suffer stressinduced diarrhea due to the inadequate development of their intestine and microbiota. ${ }^{5}$ Fermentation is considered to be an effective way to reduce the content of NSP. It has been reported that the content of cellulose and neutral detergent fiber (NDF) in wheat bran can be decreased by fermentation with rumen liquid, ${ }^{6}$ and the content of soluble dietary fibers (SDF) can be increased by sourdough-like fermentation. SDF is well-known to be largely degraded by the microbes in the hindgut of pigs, and thus is beneficial for intestinal homeostasis. $^{7}$ At present, there are few studies on the fermentation of $\mathrm{WB}$, and most microbial strains used in the existing studies are limited to a certain species of probiotics, such as Lactobacillus spp. and Bacillus subtilis. ${ }^{8,9}$ Distinguished from 
bacteria, filamentous fungi produce more abundant carbohydrate active enzymes (CAZys) with high activity, ${ }^{10}$ which have greater potential to decompose plant cell-wall polysaccharides. As reported, the cooperation of some fungi-derived CAZys, such as endoglucanase, cellobiose hydrolase and $\beta$-glucosidase, forms an efficient system called "cocktails", which shows higher activity than the sum of single enzyme. ${ }^{11}$ Therefore, we believe that the content and composition of CF in WB may be better improved by fermentation with mixed fungal strains rather than a single strain. However, there are few related studies. ${ }^{12}$ Piglets have limited tolerance to crude fiber (CF). Although the average daily gain (ADG) and average daily feed intake (ADFI) of piglets have been found to be improved by feeding 3\% WB-containing diet $\left(28.0 \mathrm{~g} \mathrm{~kg}^{-1} \mathrm{CF}\right.$ and $\left.151.0 \mathrm{~g} \mathrm{~kg}^{-1} \mathrm{NDF}\right)$ compared to a basal diet $\left(25.0 \mathrm{~g} \mathrm{~kg}^{-1}\right.$ $\mathrm{CF}$ and $\left.124.0 \mathrm{~g} \mathrm{~kg}^{-1} \mathrm{NDF}\right){ }^{13}$ the growth performance can be reduced in the case of feeding a diet with $150 \mathrm{~g} \mathrm{~kg}^{-1} \mathrm{WB}$ (42.5 $\left.\mathrm{g} \mathrm{kg}^{-1} \mathrm{CF}\right) .{ }^{14}$ Accordingly, if the content and composition of $\mathrm{CF}$ in WB are substantially improved by fermentation, the utilization of WB can be less restricted in the feed of piglets.

Briefly, we hypothesize that the nutritional value of $\mathrm{WB}$, especially the content and composition of $\mathrm{CF}$, can be improved by fermentation with mixed fungal strains. Consequently, the growth performance of pigs may not be affected, even in the case of adding a comparatively high concentration of fermented WB (FWB). If the content of SDF in FWB is improved by the fermentation as expected, the intestinal health of the pigs may also be benefited. Therefore, in the current study, raw WB was first fermented by mixed fungal strains (Aspergillus niger, Neurospora crassa, Trichoderma viride and Candida tropicalis) in the solid state and then supplemented in the diet of weaned piglets with a relatively high concentration (8\%). The growth performance, digestibility of main nutrients and intestinal health-related parameters of these animals were investigated to assess the potential of using FWB in piglets. Thus, the results of the current study provide a reference for the rational utilization of WB.

\section{Materials and methods}

\section{Fermentation, animals and diets}

The procedures for the fermentation of WB are shown in ESI $\dagger$ (1.1). After pilot fermentation, a total of 21 cross-bred (Duroc $\times$ Landrace $\times$ Yorkshire) weaned piglets with an average initial body weight (IW) of $7.20 \pm 0.50 \mathrm{~kg}$ were randomly allocated to three groups with 7 replicates per group and 1 piglet per replicate. The piglets in the control group (C) were fed a basal cornsoybean meal diet. For the diets of the WB and FWB groups, $8 \%$ of expanded corn in the basal diet was replaced by equivalent $\mathrm{WB}$ or FWB, respectively. The diets were formulated to meet the nutrient recommendations of the National Research Council (NRC, 2012) ${ }^{15}$ and no antibiotics were supplemented (Table 1). Each piglet was raised individually in a metabolism cage $(1.5 \mathrm{~m} \times 0.7 \mathrm{~m} \times 1.0 \mathrm{~m})$ and the trial lasted for 40 days. The experimental diets and water were available ad libitum
Table 1 Ingredients and nutrient composition of the diets (air-dried basis) ${ }^{a}$

\begin{tabular}{|c|c|c|c|}
\hline Item & $\mathrm{C}$ & WB & FWB \\
\hline \multicolumn{4}{|l|}{ Ingredients (\%) } \\
\hline Extruded corn, CP 7.8\% & 37.19 & 29.99 & 29.99 \\
\hline Dehulled soyabean meal, CP 47.9\% & 20.55 & 20.55 & 20.55 \\
\hline Maize starch & 18.00 & 18.00 & 18.00 \\
\hline Fishmeal, CP $60.2 \%$ & 4.50 & 4.50 & 4.50 \\
\hline Soya protein concentrate, CP $62.9 \%$ & 4.30 & 3.00 & 2.00 \\
\hline Whey powder & 5.50 & 5.50 & 5.50 \\
\hline Sucrose & 4.00 & 4.00 & 4.00 \\
\hline Glucose & 3.00 & 3.50 & 4.50 \\
\hline Soybean oil & 0.20 & 0.20 & 0.20 \\
\hline WB & - & 8.00 & - \\
\hline FWB & - & - & 8.00 \\
\hline $\mathrm{NaCl}$ & 0.30 & 0.30 & 0.30 \\
\hline L-Lys·HCl, 78.0\% & 0.40 & 0.40 & 0.40 \\
\hline DL-Met & 0.15 & 0.15 & 0.15 \\
\hline Thr, 98.5\% & 0.14 & 0.14 & 0.14 \\
\hline Trp, 98.0\% & 0.03 & 0.03 & 0.03 \\
\hline Limestone & 0.60 & 0.60 & 0.60 \\
\hline Dicalcium phosphate & 0.80 & 0.80 & 0.80 \\
\hline Chloride choline & 0.10 & 0.10 & 0.10 \\
\hline Vitamin premix ${ }^{b}$ & 0.04 & 0.04 & 0.04 \\
\hline Mineral premix ${ }^{c}$ & 0.20 & 0.20 & 0.20 \\
\hline \multirow{2}{*}{\multicolumn{4}{|c|}{ Nutrients level ${ }^{d}$}} \\
\hline & & & \\
\hline $\mathrm{DE}\left(\mathrm{Mcal} \mathrm{kg}^{-1}\right)$ & 3.53 & 3.58 & 3.58 \\
\hline DM (\%) & 97.28 & 96.44 & 96.47 \\
\hline $\mathrm{CP}(\%)$ & 17.45 & 17.37 & 16.30 \\
\hline EE $(\%)$ & 2.10 & 2.32 & 1.99 \\
\hline Crud ash (\%) & 5.07 & 5.10 & 5.33 \\
\hline CF (\%) & 1.92 & 4.18 & 4.11 \\
\hline $\mathrm{NDF}(\%)$ & 12.12 & 15.22 & 14.12 \\
\hline $\operatorname{ADF}(\%)$ & 10.61 & 13.95 & 12.17 \\
\hline SDF (\%) & 2.10 & 2.21 & 3.96 \\
\hline IDF (\%) & 7.83 & 9.42 & 8.47 \\
\hline D-Lys (\%) & 1.44 & 1.26 & 1.31 \\
\hline D-Met (\%) & 0.45 & 0.58 & 0.42 \\
\hline D-Met + D-Cys (\%) & 0.66 & 0.77 & 0.66 \\
\hline D-Thr (\%) & 0.86 & 0.79 & 0.79 \\
\hline D-Trp (\%) & 0.28 & 0.29 & 0.20 \\
\hline $\mathrm{Ca}(\%)$ & 1.00 & 0.99 & 0.79 \\
\hline ТP (\%) & 0.63 & 0.60 & 0.55 \\
\hline $\mathrm{AP}(\%)$ & 0.43 & 0.42 & 0.40 \\
\hline
\end{tabular}

${ }^{a} \mathrm{C}$, control; WB, wheat bran; FWB, fermented wheat bran; CP, crude protein; DE, digestible energy; EE, ether extract; $\mathrm{CF}$, crude fiber; NDF, neutral-detergent fiber; ADF, acid-detergent fiber; SDF, soluble dietary fiber; IDF, Insoluble dietary fiber; Ca, calcium; TP, total phosphorus; and AP, available phosphorus. ${ }^{b}$ The vitamin premix provided: Vitamin A 30000000 IU; Vitamin $D_{3} 10000000$ IU; Vitamin E 80000 IU; Vitamin $\mathrm{K}_{3} 10000 \mathrm{mg}$; Vitamin $\mathrm{B}_{1} 10000 \mathrm{mg}$; Vitamin $\mathrm{B}_{2} 25000 \mathrm{mg}$; Vitamin $\mathrm{B}_{6} 12000 \mathrm{mg}$; Vitamin $\mathrm{B}_{12} 120 \mathrm{mg}$; D-pantothenic acid $50000 \mathrm{mg}$; folic acid $5000 \mathrm{mg}$; and biotin $500 \mathrm{mg}$ per $\mathrm{kg}$ of diet. ${ }^{c}$ The mineral premix $(7-25 \mathrm{~kg})$ provided: $350.00 \mathrm{mg} \mathrm{Fe}\left(\mathrm{FeSO}_{4} \cdot \mathrm{H}_{2} \mathrm{O}\right)$; $41.67 \mathrm{mg} \mathrm{Cu}\left(\mathrm{CuSO}_{4} \cdot 5 \mathrm{H}_{2} \mathrm{O}\right) ; 292.78 \mathrm{mg} \mathrm{Zn}\left(\mathrm{ZnSO}_{4} \cdot 7 \mathrm{H}_{2} \mathrm{O}\right) ; 66.20 \mathrm{mg} \mathrm{Mn}$ $\left(\mathrm{MnSO}_{4} \cdot \mathrm{H}_{2} \mathrm{O}\right) ; 8.31 \mathrm{mg} \mathrm{I}(\mathrm{KI}) ; 30.61 \mathrm{mg}$ Se $\left(\mathrm{Na}_{2} \mathrm{SeO}_{3}\right) ; 1209.55 \mathrm{mg}$ $\mathrm{CaCO}_{3}$ per kg of diets. ${ }^{d}$ The level of DE, CP, EE, CF, NDF, ADF, SDF, IDF and crude ash was the measured value, while the content of $\mathrm{Ca}$, AP, TP, D-Lys, D-Met, D-Met + D-Cys, D-Thr and D-Trp was the calculated value.

during the entire experimental period. The feed intake and general health of each piglet were recorded daily. The ADG, ADFI and feed gain ratio $(F / G)$ for each pig were then calculated at the end of the experiment. The fecal sample from each piglet was scored as follows: 0 , normal; 1 , pasty; 2 , semi-liquid; 
and 3, liquid. Piglets with a daily fecal score of $\geq 2$ were considered as suffering from diarrhea. The diarrhea rate was calculated as follows:

$$
\begin{aligned}
& \text { Diarrhea rate }(\%)=(\text { Total number of pigs per pen with diarrhea }) / \\
& (\text { Number of pigs per pen } \times n) / 100
\end{aligned}
$$

where $n$ represents the experimental duration in days.

\section{Sample collection}

The feces from all piglets were collected continuously for $12 \mathrm{~h}$ from $08: 00 \mathrm{~h}$ to $20: 00 \mathrm{~h}$ on day 37 to 40 during the trial for the determination of nutrient digestibility. For each collected fecal and diet sample, $10 \%$ hydrochloric acid was added for the fixation of excreta nitrogen. Then all samples were dried and finely ground to analyze the dry matter (DM), gross energy (GE), crude protein (CP), crude fiber (CF), neutral-detergent fiber (NDF), acid-detergent fiber (ADF), soluble dietary fiber (SDF), insoluble dietary fiber (IDF) and acid insoluble ash (AIA). At the end of the trial, all the piglets were weighed and $10 \mathrm{~mL}$ jugular blood was collected from each pig for the determination of $\mathrm{T}$ lymphocyte subsets $(8 \mathrm{~mL}$ with $2 \mathrm{~mL}$ heparin sodium solution) and biochemical analysis $(2 \mathrm{~mL})$. Subsequently, each piglet was anaesthetised by a lethal injection of sodium pentobarbital ( $200 \mathrm{mg}$ per $\mathrm{kg}$ body weight) and its abdomen was immediately opened. The middle section (approximate $2 \mathrm{~cm}$ ) of the duodenum, jejunum, ileum and colon of each pig was collected and fixed in $100 \mathrm{~mL}$ of $10 \%$ formaldehyde solution for the following histology analysis. The mucosa of each intestinal segment from each pig was scraped using a sterilized slide and immediately put in liquid nitrogen for the following RNA extraction. Another $10 \mathrm{~g}$ of the colonic digesta from each piglet was collected and kept in liquid nitrogen for the quantification of microbial species and the measurement of short chain fatty acids (SCFAs). Approximately $5 \mathrm{~g}$ of spleen was also collected for the determination of $\mathrm{T}$ lymphocyte subsets.

\section{Determination of apparent total-tract nutrient digestibility}

The apparent total-tract digestibility of the main nutrients was measured using a method with acid-insoluble ash (AIA) as an indicator. The AIA in the diet and fecal samples was determined using the method described by Chinese National Standard (GB/T23742). The determination of DM, GE, CP, CF, $\mathrm{NDF}, \mathrm{ADF}, \mathrm{SDF}$ and IDF is described in detail in the ESI. $\dagger$ The apparent total-tract digestibility of the nutrients was calculated according to the described method. ${ }^{16}$

\section{The histological analysis of the intestine, and the determination of serum biochemical parameters and $T$ lymphocyte subsets in the blood and spleen}

Each collected intestinal segment saved in formaldehyde solution was prepared using a standard paraffin-embedding method. ${ }^{17}$ About 5 mm-thick cross-sections of each sample were stained with hematoxylin and eosin, and then investigated under a $100 \times$ magnified view with an Olympus CKX
53 microscope (Japan). To measure the height of villous (HV) and the depth of crypt (DC), the prepared slices were observed and photographed. For each image (one slice), a total of 5 nondestructive fields were selected, and the HV and CD of each field were recorded. The average value of the 5 fields was calculated as the final data. The ratio of HV/DC was calculated as VCR. For each sample, the number of positively stained goblet cells on five randomly selected villi was counted using the Image-Pro Plus software (version 6.0, Media Cybernetics) and the average number was calculated. The concentration of D-lactate and lipopolysaccharide (LPS) in the serum of each piglet was assayed using a porcine D-lactate and porcine lipopolysaccharide ELISA kit purchased from Wuhan Meimian Biological Technology Co., Ltd (Hubei, China), respectively. The determination of $\mathrm{T}$ lymphocyte subsets in the jugular blood and spleen is described in detail in the ESI† (1.2).

\section{RNA extraction and real-time PCR analysis}

The total RNA from approximately $0.1 \mathrm{~g}$ mucosal sample from each piglet was extracted using Trizol (TAKARA, Japan), and then reversely transcribed into cDNA using the PrimeScriptTM RT reagent kit (TAKARA, Japan). The $10 \mu \mathrm{L}$ real-time PCR reaction mixture included a $1.0 \mu \mathrm{L}$ cDNA template, $5 \mu \mathrm{L}$ SYBR Green mix, $0.4 \mu \mathrm{L}$ each of the forward and reverse primers, $0.2 \mu \mathrm{L}$ correction fluid ROX (TAKARA, Japan) and $3 \mu \mathrm{L}$ double distilled water. The PCR reaction (ABI 7900, USA) for each gene was conducted as follows: an initial step at $95{ }^{\circ} \mathrm{C}$ for $30 \mathrm{~s}$, followed by 40 cycles of denaturation at $95{ }^{\circ} \mathrm{C}$ for $5 \mathrm{~s}$, annealing at $60{ }^{\circ} \mathrm{C}$ for $34 \mathrm{~s}$, and extension at $72{ }^{\circ} \mathrm{C}$ for $15 \mathrm{~s}$. Three housekeeping genes, $\beta$-actin, $18 s$ rRNA and GAPDH, were selected as internal references, and the relative expression of each target gene was calculated using the $2^{-\Delta \Delta \mathrm{Ct}}$ method. ${ }^{18}$ The sequences of primers, the length of the PCR products and the corresponding references are shown in ESI Table S1.†

\section{DNA extraction, quantification of bacteria and measurement of SCFAs in the colonic digesta samples}

The methods for the extraction of genomic DNA and the measurement of SCFAs (acetate, propionate, butyrate, isobutyrate, valerate, and isovalerate) in the digesta samples were described in our previous study. ${ }^{7}$ The copy numbers of total bacteria, Bifidobacterium, Lactobacillus, Clostridium cluster IV, Clostridium cluster XIVa, butyrate-producing bacteria (Fecalibacterium prausnitzii, Eubacterium rectale, Clostridium butyricum and Anaerostipes caccae), E. coli, Salmonella spp., Enterobacteriaceae family, Streptococcus and the three genes encoding the virulence factors of enterotoxic E. coli, Heatstable toxin I (STa), Heat-stable toxin II (STb) and Aerobactin enteroaggregative toxin (astA), in each sample were quantified by real-time PCR on a Bio-RadCFX96 real-time system (BioRad, USA) using SYBR Green as the fluorescent dye. The information for the primers is shown in ESI Table S1.†

\section{Statistical analysis}

All data was firstly checked for normal distribution using the descriptive statistic (Explore) module of the SPSS 22.0 software. 
For the data with a normal distribution, one-way ANOVA was used to analyze the difference among the groups, and the homogeneity of variance among the groups was tested using Duncan's multiple-range tests. The data is presented as the mean with the pooled standard error (SE). In the case of the skewed data, Kruskal-Wallis nonparametric test was used to analyze the difference among groups, and the values represent media with the quantification of bacteria (IQR). Differences were considered to be significant when $P<0.05$ and insignificant when $P \geq 0.05$. The correlation between the butyrate concentration and bacterial species was calculated using Pearson's correlational analysis and the results were visualized using the vegan, ggcor and dplyr packages in $\mathrm{R}$ 4.0.1.

\section{Results}

\section{Comparison of nutrients and microstructure in WB and FWB}

Compared with WB, the level of DM, GE, EE, CP and SDF in FWB increased, while the level of CF, NDF and ADF decreased (Table S2†). According to the scanning electron microscopy (SEM) images, the surface of the WB particle exhibited a complete and regular morphology (Fig. 1A), whereas the outer layer of the FWB particle was partially fractured with a slightly inhomogeneous pore structure inside (Fig. 1B).

\section{Effect of WB and FWB on the growth performance and} nutrient digestibility in weaned piglets

Compared with the control, the ADG of WB and FWB pigs increased by 8.78 and $6.36 \mathrm{~g} \mathrm{~d}^{-1}(P>0.05)$, respectively (ESI Table S3 $\dagger$ ). The digestibility of CP and EE in the WB pigs, and the digestibility of EE and CF in the FWB pigs increased $(P<$ 0.05 ) compared to the control (Table 2), while the digestibility of $\mathrm{NDF}$ and $\mathrm{ADF}$ in the $\mathrm{WB}$ pigs decreased $(P<0.05)$. In addition, the digestibility of NDF and ADF in the FWB pigs was increased compared to the WB pigs $(P<0.05)$.
Effect of WB and FWB on the diarrhea rate and intestinal morphology in weaned piglets

The diarrhea rate of the WB pigs during 5-10 d, 20-25 d and 30-35 d, together with that of FWB pigs during 20-25 d and 30-35 d decreased compared to the control $(P<0.05$, ESI Fig. S1†). According to the results of the histological analysis, no difference was found in the HV, CD, VCR and number of goblet cells in the duodenum and ileum of the pigs among the groups $(P>0.05$, Fig. 2A and $\mathrm{C})$. However, the $\mathrm{CD}$ of the jejunum in the FWB pigs was lower than control $(P<0.05$, Fig. 2B), but the VCR of the jejunum in the WB and FWB piglets increased $(P<0.01)$. In addition, the number of goblet cells in the jejunum of the FWB pigs increased compared to was WB pigs $(P<0.05$, Fig. $2 \mathrm{~B})$.

Effect of WB and FWB on the serum biochemical indices and $T$ lymphocyte subsets in the spleen and blood of weaned piglets

The level of serum D-lactate and LPS was measured to show the intestinal permeability of the piglets (ESI Fig. S2 $\dagger$ ), and no statistical significance was found in both two parameters among the groups $(P>0.05)$.

According to the results of the flow cytometric analysis (ESI Table $\mathrm{S} 4 \dagger$ ), the percentage of $\mathrm{CD}^{+} \mathrm{T}$ lymphocytes in the spleen of the WB piglets decreased compared to the FWB and control pigs $(P<0.01)$, while it was higher in the blood of the FWB piglets than that of the WB and control pigs $(P<0.01)$. Additionally, the percentage of $\mathrm{CD}^{+} \mathrm{T}$ lymphocytes in the blood of the FWB piglets was also higher than that of the WB piglets $(P<0.05)$.

Effect of WB and FWB on the expression of intestinal barrier and inflammatory response-related genes in the weaned piglets

Compared with the control, the mRNA level of MUC1 and $p B D 1$ in the jejunal mucosa of the FWB piglets $(P<0.05$, Fig. 3A), $p B D 1$ in the colonic mucosa of the $\mathrm{WB}$ and FWB
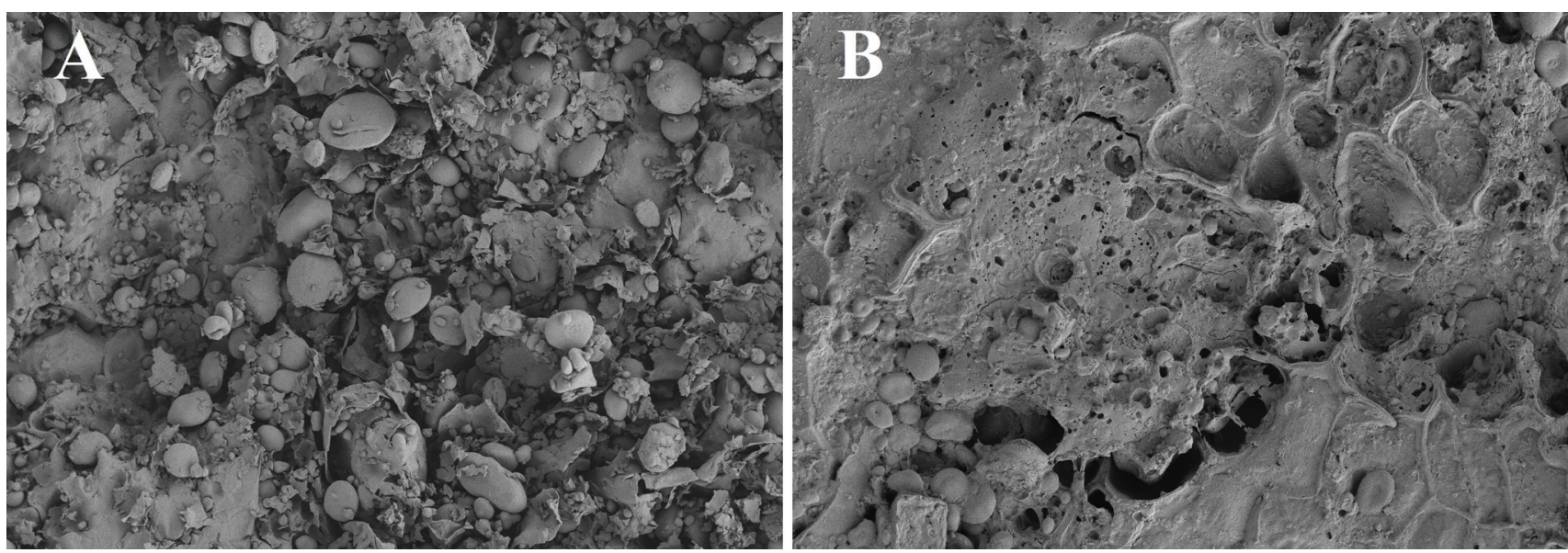

Fig. 1 SEM images of WB (wheat bran) and FWB (fermented wheat bran). (A) WB and (B) FWB. 
Table 2 The digestibility of nutrients (\%) in piglets ${ }^{a, b}$

\begin{tabular}{|c|c|c|c|c|c|c|c|c|}
\hline \multirow[b]{2}{*}{ Item } & \multicolumn{3}{|l|}{ Group } & \multirow[b]{2}{*}{ Pooled SE } & \multicolumn{4}{|l|}{$P$-Value } \\
\hline & $\mathrm{C}$ & WB & FWB & & Group & C vs. WB & C vs. FWB & WB vs. FWB \\
\hline Energy & 86.13 & 88.78 & 88.1 & 0.68 & 0.12 & 0.05 & NS & NS \\
\hline $\mathrm{DM}$ & 86.34 & 88.87 & 87.99 & 0.55 & 0.15 & 0.06 & NS & NS \\
\hline $\mathrm{CP}$ & $74.84^{\mathrm{b}}$ & $81.08^{\mathrm{a}}$ & $79.92^{\mathrm{ab}}$ & 1.23 & 0.07 & 0.03 & 0.09 & NS \\
\hline $\mathrm{EE}$ & $39.87^{\mathrm{a}}$ & $58.70^{\mathrm{b}}$ & $52.85^{\mathrm{b}}$ & 2.46 & $<0.01$ & 0.00 & 0.01 & NS \\
\hline $\mathrm{CF}$ & $58.28^{\mathrm{a}}$ & $62.58^{\mathrm{ab}}$ & $67.13^{b}$ & 1.58 & 0.07 & NS & 0.02 & NS \\
\hline NDF & $72.30^{\mathrm{a}}$ & $64.65^{\mathrm{b}}$ & $73.68^{\mathrm{a}}$ & 2.29 & $<0.01$ & NS & 0.01 & $<0.01$ \\
\hline $\mathrm{ADF}$ & $80.29^{a}$ & $72.29^{b}$ & $82.32^{\mathrm{a}}$ & 1.87 & $<0.01$ & NS & $<0.01$ & $<0.01$ \\
\hline
\end{tabular}

${ }^{a}$ Normally distributed data is presented as mean, while non-normally distributed data is presented as median $(n=7)$. SE, standard error of mean; NS, not significant; C, control; WB, wheat bran; and FWB, fermented wheat bran. The variant alphabetical superscript in the same row indicates significant difference $(P<0.05, n=7)$. Among the data, the digestibility of NDF and ADF is non-normally distributed. ${ }^{b}$ DM, dry matter; $\mathrm{CP}$, crude protein; EE, ether extract; $\mathrm{CF}$, crude fiber; NDF, neutral detergent fiber; and ADF, acid detergent fiber.

A
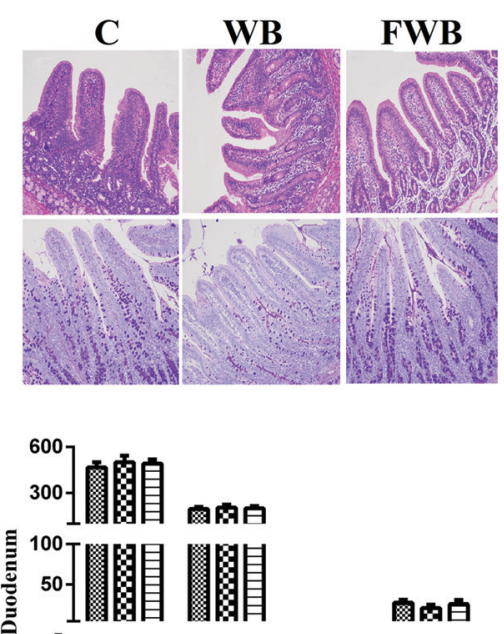

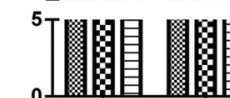

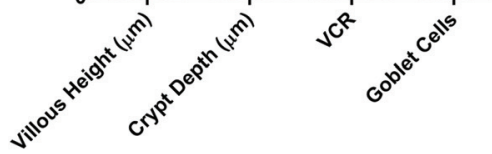

B
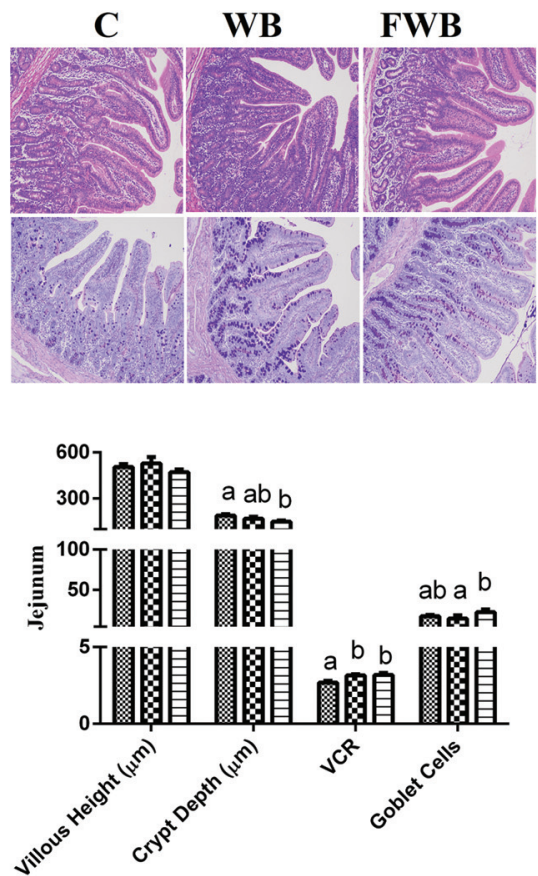

$\mathrm{ab} a \mathrm{~b}$

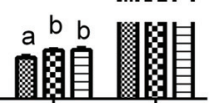

$\mathbf{C}$
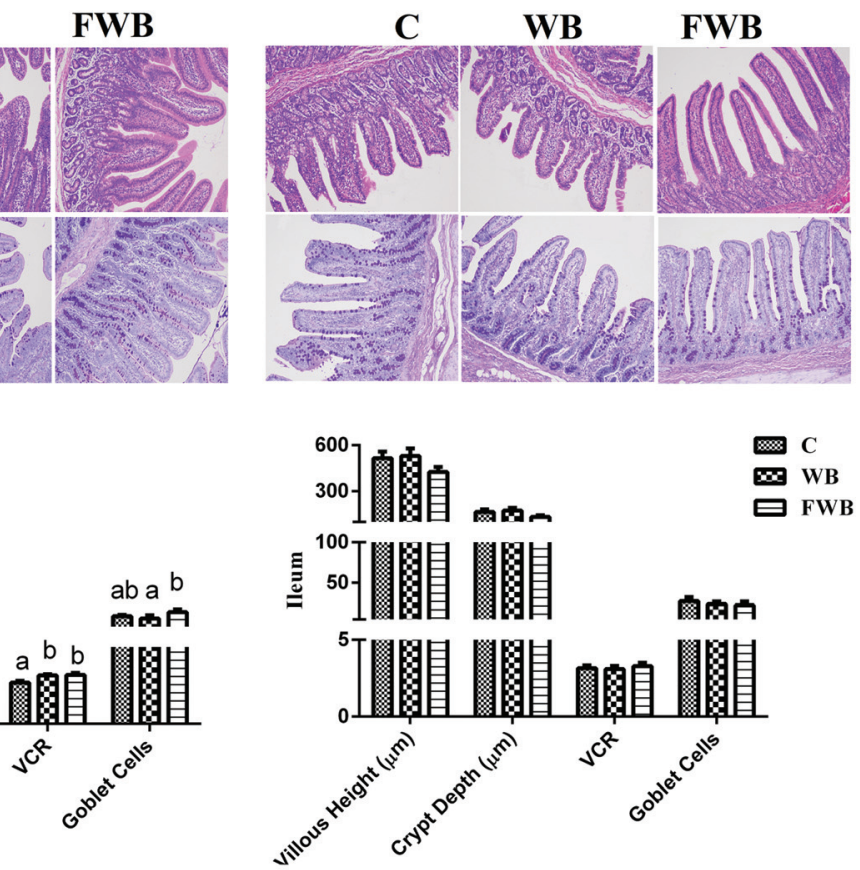

Fig. 2 Morphology of intestinal villi $(H E ; \times 100)$ and number of goblet cells (PAS; $\times 100)$ in different intestinal segments of piglets. (A) Villous height, crypt depth, VCR and number of goblet cells in the duodenum of piglets in different groups. (B) Villous height, crypt depth, VCR and number of goblet cells in the jejunum of piglets in the different groups. (C) Villous height, crypt depth, VCR and number of goblet cells in the ileum of piglets in the different groups. VCR height villous/depth crypt ration. C, control; WB, wheat bran; and FWB, fermented wheat bran.

piglets $(P<0.05$, Fig. 3B), and $I L-10$ in the jejunal mucosa of the WB piglets $(P<0.05$, Fig. 3C) increased.

Effect of WB and FWB on the quantity of bacterial groups and the concentration of SCFAs in the colonic digesta of weaned piglets

The results of real-time PCR showed that the copies of total bacteria, Clostridium cluster IV, Fecalibacterium prausnitzii, Clostridium butyricum and Anaerostipes caccae, in the colonic digesta of the WB piglets, and the copies of Eubacterium rectale in the WB and FWB piglets increased compared to the control
$(P<0.05$, Fig. 4A). Meanwhile, the copies of Streptococcus in $\mathrm{WB}$ and FWB, and $S T b$, one of the virulence factors of $E$. coli, in the FWB pigs decreased compared to the control $(P<0.05$, Fig. 4B).

According to the results of gas chromatography (Fig. 4C), the concentration of butyrate in the colonic digesta of WB and FWB piglets, as well as the concentration of total SCFAs in WB piglets showed higher than pigs in control group $(P<0.05)$.

Furthermore, Pearson's correlation analysis (Fig. 4D) showed positive correlations $(P<0.01$ or 0.05$)$ between the copies of certain bacterial groups and the concentration of 
A

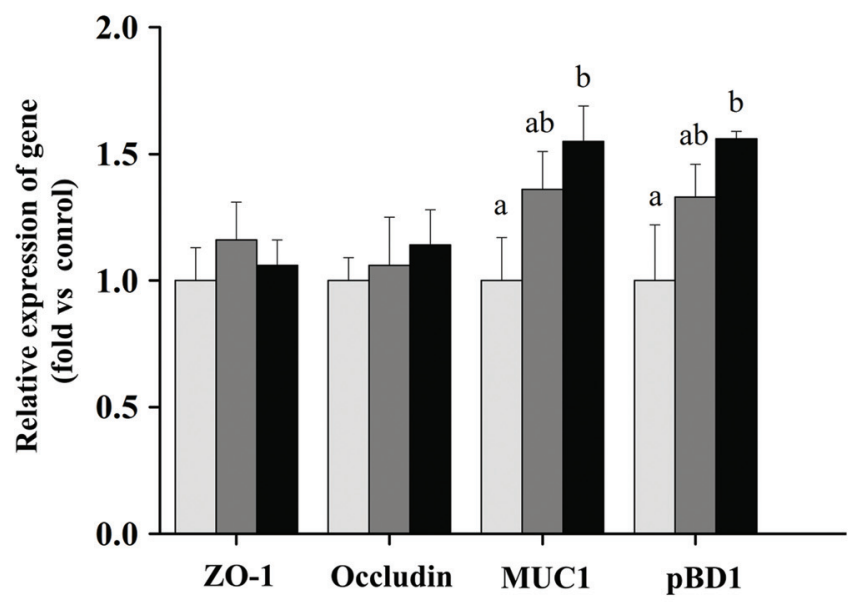

C

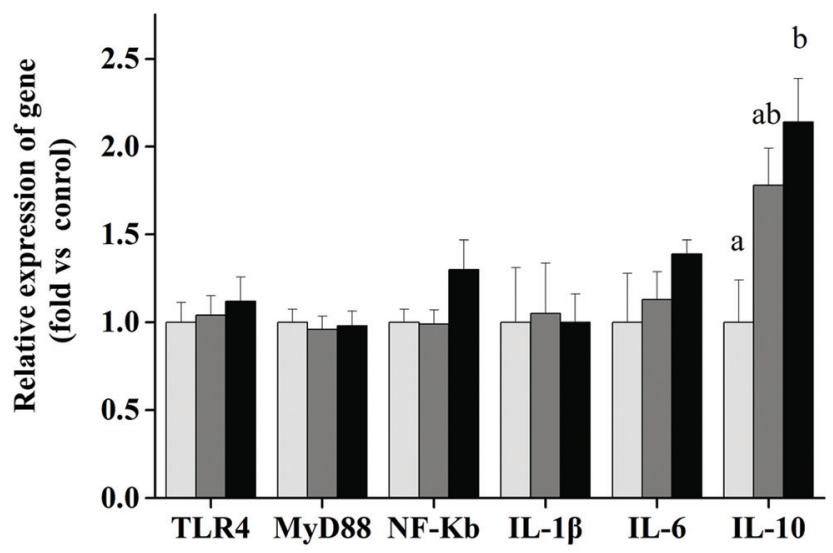

B

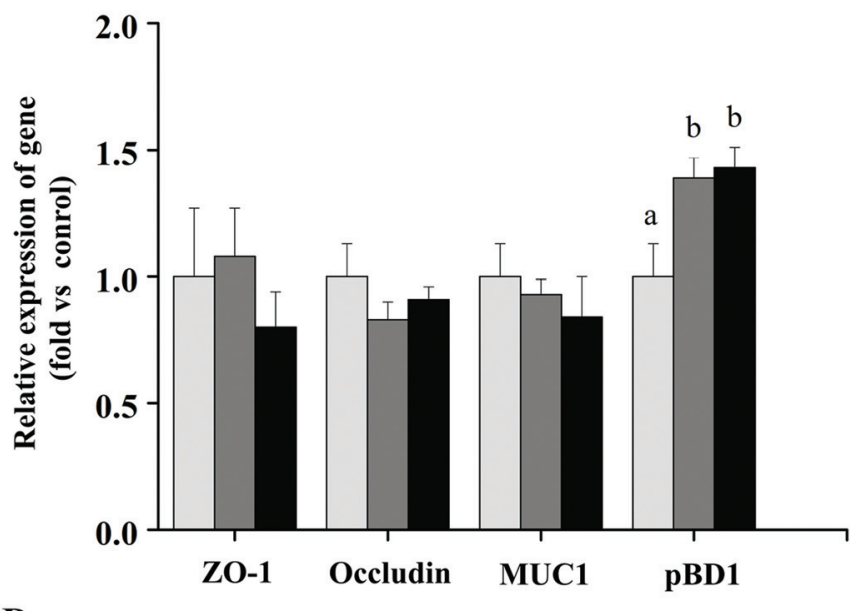

D

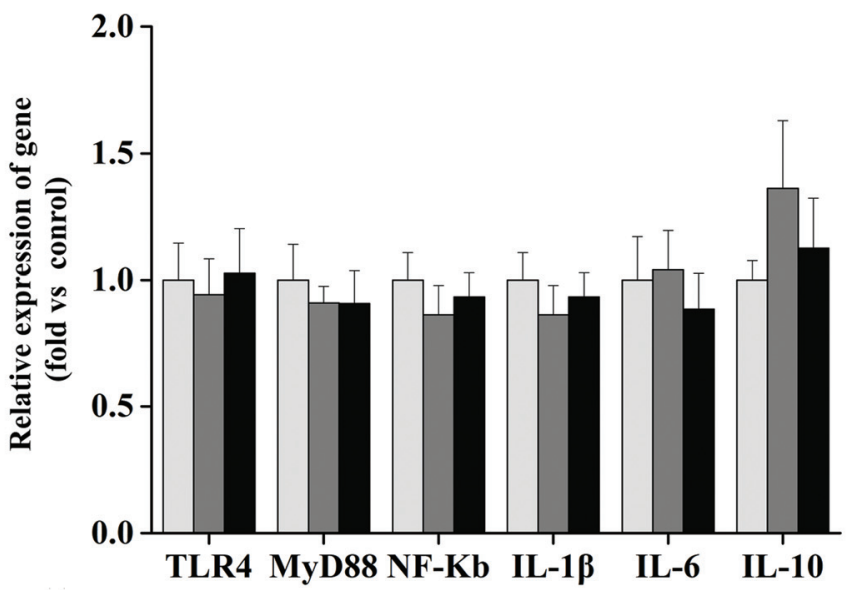

Fig. 3 Relative mRNA level of genes related to intestinal barrier and inflammatory cytokines in the jejunal and colonic mucosa of piglets in the different groups $(n=7)$. Superscripts of lowercase letters represent significant differences $(P<0.05)$. (A) Relative expression of genes related to mucosal barrier in the jejunal mucosa of piglets. (B) Relative expression of genes related to mucosal barrier in the colonic mucosa of piglets. (C) Relative expression of genes related to inflammatory cytokines in the jejunal mucosa of piglets. (D) Relative expression of genes related to inflammatory cytokines in the colonic mucosa of piglets. C, control; WB, wheat bran; and FWB, fermented wheat bran.

SCFAs. In particular, the number of Lactobacillus, Bifidobacterium, Clostridium XIVa, Fecalibacterium prausnitzii, Eubacterium rectale, Clostridium butyricum and Anaerostipes caccae was positively $(P<0.01$ or 0.05$)$ correlated with the concentration of butyrate in the colonic digesta of the piglets.

\section{Discussion}

It has been found that the supplement of wheat bran in the diet can effectively alleviate diarrhea and promote the intestinal health of weaned piglets, ${ }^{19}$ which can probably be attributed to the special physiological function of $\mathrm{DF}$ in $\mathrm{WB} .^{20}$ However, the high level of NSP in WB also increases the feed conversion rate and reduces the growth performance of pigs, ${ }^{2}$ especially when supplemented with a relatively high concentration in the feed. Furthermore, the nutritional and physiological effects of WB largely depend on the composition of DF, such as the content of SDF or IDF. For instance, the proliferation of pathogens can be inhibited by IDF by decreasing the transit time of digesta in the gut, ${ }^{21}$ while SDF is regarded as one of the preferable substrates for gut microbes to produce SCFAs, which play an important role in the glycolipid metabolism and gut health of the host. ${ }^{22-24}$ Although fermentation is considered to be an effective strategy to reduce the content of CF in plant-derived feedstuffs, few studies have focused on the comparison of the effects of high-level WB and FWB in weaned piglets, which was particularly discussed in the current study.

The improvement of nutritional values in fermented feedstuffs largely depends on the microbial strains. NDF in WB has been proven to be decreased by $5.6 \%$ with the fermentation of fungi such as Trichoderma, with a $1.5 \%$ decrease in $\mathrm{ADF}$ and a $3.4 \%$ increase in $\mathrm{CP}^{25}$ In this study, the content of $\mathrm{DM}, \mathrm{CP}(+7.7 \%)$, EE and SDF (+62.9\%) in WB increased by the fermentation of mixed fungal strains, and the content of $\mathrm{CF}$ 
A

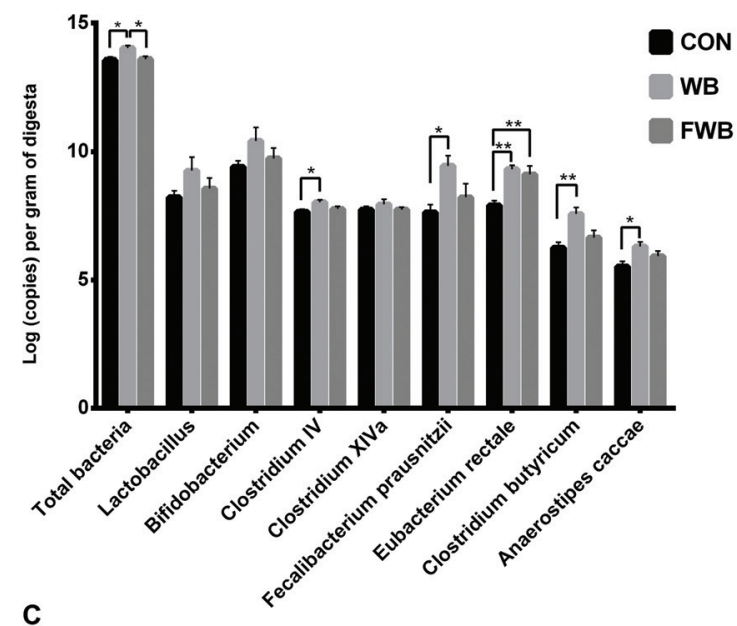

C

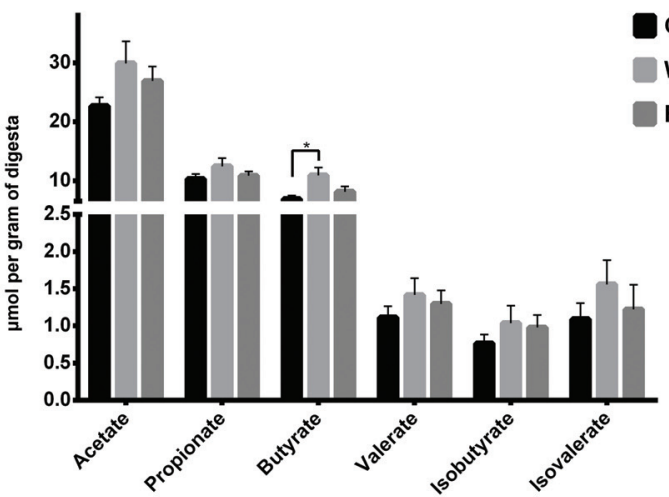

B
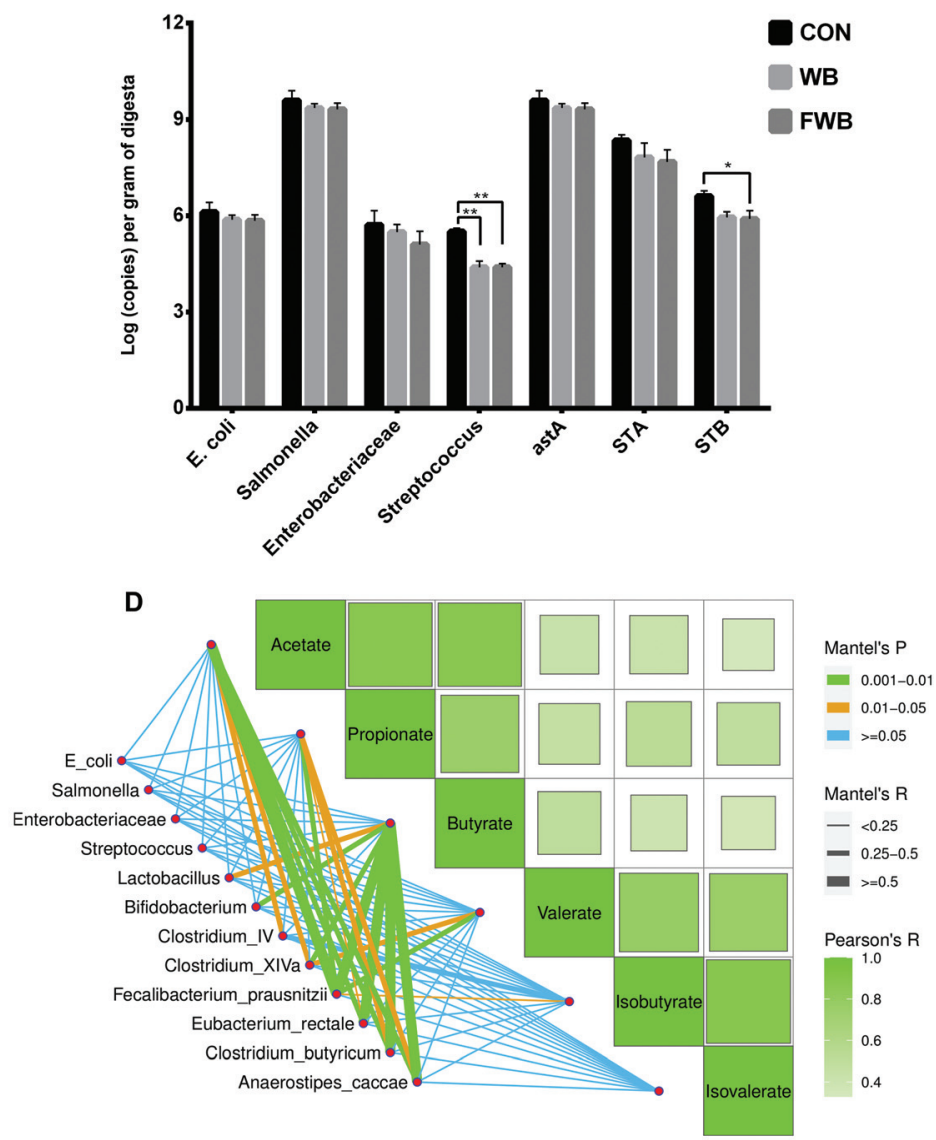

Fig. 4 Quantity of bacterial groups, concentrations of SCFAs, and correlation between the number of bacteria and SCFA concentration in the colonic digesta of the weaned pigs. (A) Copies of total bacteria, beneficial bacterial groups and main butyrate-producing bacteria. (B) Copies of conditioned pathogens and virulence factors. (C) Concentrations of SCFAs. (D) Pearson's correlation between the concentration of SCFAs and bacterial

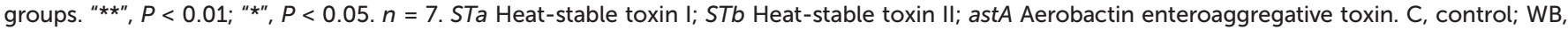
wheat bran; FWB, and fermented wheat bran.

$(-24.5 \%), \mathrm{NDF}(-12.2 \%), \mathrm{ADF}(-23.6 \%)$ and $\mathrm{IDF}(-7.8 \%)$ was reduced as expected. This significant improvement in CF than the previous study highlights the advantages of mixed filamentous fungi over a single strain. Interesting results were found when FWB was applied to weaned piglets in a high (8\%) concentration. Although the measured concentration of CF in the WB $(4.18 \%)$ and FWB $(4.11 \%)$ diets were higher than the basal diet $(1.92 \%)$, only the digestibility of CF in the piglets fed FWB was remarkably improved. An improvement in the digestibility of DM, CF and EE was also observed in the growing pigs fed $20 \%$ FWB. $^{26}$ These findings suggest the potential of FWB in improving the nutrient digestibility, especially $\mathrm{CF}$, in weaned pigs even with a higher concentration in the feed.

Stress-induced diarrhea is a major risk factor for the gut health of weaned piglets. The supplement of WB has been reported to relieve the diarrhea of post-weaning pigs due to a reduction in pathogenic $E$. coli. ${ }^{17}$ In this study, we did not investigate the significant decrease in the whole-stage diarrhea rate in pigs fed WB and FWB compared to the control.
However, the copies of Streptococcus and/or E. coli enterotoxin $(S T b)$ decreased in these animals with a decline in the phased diarrhea rate. This may be due to the "broom-like" characteristic of DF, which has been found to be critical for the cleaning of pathogens and promoting the development of intestinal villi. ${ }^{27}$ Indeed, the VCR of the jejunum in the pigs fed WB and FWB was found to be improved. Our previous study also showed that the morphology of the ileum in finishing pigs can be improved by WB ranging from 100 to $300 \mathrm{~g} \mathrm{~kg}^{-1} .^{28}$

Intestinal epithelial cells (IECs), endocrine cells, goblet cells and Paneth cells existing in the intestinal villi constitute to the main part of the epithelial barrier. ${ }^{29}$ Tight junctions (TJs) between IECs, mucin secreted by goblet cells and antimicrobial peptides (AMPs) are the three important factors involved in the barrier function. ${ }^{25}$ In the current study, both the expression of $M U C 1$ and $p B D 1$ in the jejunal mucosa of pigs fed FWB was markedly increased compared to the control, and the number of goblet cells in these pigs was higher than that in the pigs fed WB. This may be attributed to 
the higher content of SDF in FWB. High-viscosity SDF has been reported to increase the number of goblet cells and upregulate the expression of MUC1 in the small intestine of rats. ${ }^{30}$ A diet with $23 \%$ beet residue enriched in SDF was also proven to increase the number of goblet cells in the jejunum of weaned piglets. ${ }^{31}$ In pigs, pBD1 secreted by Paneth cells is one of the natural AMPs to prevent the adhesion of pathogenic microbes to mucosa. ${ }^{32,33}$ A large number of studies present the regulation of SCFAs, particularly butyrate, in the expression of $p B D$. In vitro, sodium butyrate enhances the expression of pBD2 and $p B D 3$ in IECs, macrophage and monocytes. ${ }^{34}$ In vivo, butyrate relieves the intestinal inflammation in $E$. coli-infected piglets via the regulation of $p B D$ expression, and the further inhibition of histone deacetylase. ${ }^{35}$ In the current study, the concentration of butyrate in the colon of pigs fed WB truly increased compared to the control, which can be reasonably inferred to be associated with the increased expression of $p B D$. In addition, butyrate is the most important energy source of IECs, which plays a key role in promoting cell differentiation and maturation and maintaining the intestinal homeostasis. ${ }^{42}$ It is also the main metabolite of butyrate-producing bacteria using complex carbohydrates such as SDF. ${ }^{36}$ The Pearson's correlation analysis proved that the elevated butyrate in the colon of the WB pigs is attributed to the increase in the number of butyrate-producing bacteria such as Fecalibacterium, Clostridium and Anaerostipes. Interestingly, although the content of SDF in FWB was higher than that in WB, the butyrate concentration in the colon of the FWB pigs was lower than that in the WB pigs. However, we indeed observed a marked increase in Eubacterium rectale, a well-known butyrate-producing bacterium, in the colon of the FWB pigs compared to control. This is probably due to the larger differences between the individuals in the FWB group.

The proportion of different $\mathrm{T}$ lymphocyte subsets can be used to reflect the systemic immunity of animals. ${ }^{37}$ CD3 is a class of receptors that can be widely found in all $\mathrm{T}$ lymphocytes for the recognition of antigens. ${ }^{38}$ CD4 induces T helper cells to enhance the humoral immune response and secrete inflammation-associated cytokines such as IL-4, IL-6 and IL-10 ${ }^{39,40}$ mediated by signal pathways related to pattern recognition receptors such as TLR4/MyD88/NF-кB. ${ }^{41}$ We observed an increase in $\mathrm{CD}^{+}$(vs. WB and control) and $\mathrm{CD} 4^{+}$(vs. WB) $\mathrm{T}$ lymphocytes in the blood of the pigs fed FWB, which is consistent with the increased expression of $I L-10$ in the jejunal mucosa. However, there was no remarkable change in the expression of $T L R 4, M y D 88$ and $N F-\kappa B$, indicating that this increased expression of $I L-10$ may not be mediated by the TLR4/MyD88/NF- $\kappa$ B pathway.

\section{Conclusion}

In summary, herein, we developed a mixed fungi-fermented WB with a comparatively higher content of DM, CP and SDF, and a lower content of CF, NDF, ADF and IDF, which increased the digestibility of NDF and ADF in weaned pigs compared to
$\mathrm{WB}$, even with a high concentration $(8 \%)$ in the feed. Both the dietary supplement of WB and FWB decreased the number of conditional pathogens such as Streptococcus or/and E. coli virulence factor (STb). FWB was better than WB in improving the number of abundant goblet cells, the expression of MUC1 and anti-inflammatory cytokine $(I L-10)$ in the jejunal mucosa, and the proportion of blood $\mathrm{CD}^{+}{ }^{-} \mathrm{T}$ lymphocyte subset. The concentration of butyrate in the colon of the weaned pigs was promoted by the supplement of WB rather than FWB, but both the supplement WB and FWB increased the number of butyrate-producing bacteria. Overall, both the supplement of $8 \%$ WB and FWB did not affect the growth performance of the weaned pigs. Thus, compared with WB, our newly developed FWB showed an obvious advantage in improving the digestibility of fiber in weaned pigs.

\section{Availability of data and materials}

All data generated or analyzed during the study are included in this published article.

\section{Author contributions}

YL designed the experiment and wrote the manuscript. JH and HL performed animal trial and data analysis. CL and JC helped with the animal trial and sample collection. JC, HC, GT, HW, QW and JH helped with the data analysis. DC, BY, $\mathrm{ZH}, \mathrm{PZ}$ and XM helped with the writing. JY, JL, AW and HY helped with the revision of the manuscript.

\section{Consent for publication}

Not applicable.

\section{Ethics approval and consent to participate}

All experimental procedures and animal care were accomplished in accordance with the Guide for the Care and Use of Laboratory Animals provided by the Institutional Animal Care Advisory Committee for Sichuan Agricultural University. The experimental protocols used in the present study were approved by the Sichuan Agricultural University Institutional Animal Care and Use Committee No. 69130079.

\section{Conflicts of interest}

The authors state that there are no conflicts of interest. 


\section{Acknowledgements}

This study was supported by National Natural Science Foundation of China (31872369, 32072743) and the "Double Support Plan” Foundation of Sichuan Agricultural University.

\section{References}

1 M. M. Javed, Wheat bran as a brown gold: Nutritious value and its biotechnological applications, Afr. J. Microbiol. Res., 2012, 6, 724-733.

2 L. Stevenson, F. Phillips, K. O'Sullivan and J. Walton, Wheat bran: its composition and benefits to health, a European perspective, Int. J. Food Sci. Nutr., 2012, 63, 10011013.

3 O. A. H. Simmins, Future of NSP-degrading enzymes to improve nutrient utilization of co-products and gut health in pigs, Livest. Sci., 2010, 134, 255-257.

4 M. S. Chandra, B. Viswanath and B. R. Reddy, Optimization of extraction of $\beta$-endoglucanase from the fermented bran of Aspergillus niger, Indian J. Microbiol., 2010, 50, 122-126.

5 L. Montagne, G. Boudry, C. Favier, I. L. Huerou-Luron, J. P. Lalles and B. Seve, Main intestinal markers associated with the changes in gut architecture and function in piglets after weaning, Br. J. Nutr., 2007, 97, 45-57.

6 M. R. Debi, B. A. Wichert and A. Liesegang, Method development to reduce the fiber content of wheat bran and rice bran through anaerobic fermentation with rumen liquor for use in poultry feed, Asian-Australas. J. Anim. Sci., 2018, 32, 395-404.

7 Y. H. Luo, L. Zhang, H. Li, H. Smidt, A. D. G. Wright, K. Y. Zhang, X. M. Ding, Q. F. Zeng, S. P. Bai, J. P. Wang, J. Li, P. Zheng, G. Tian, J. Y. Cai and D. W. Chen, Different types of dietary fibers trigger specific alterations in composition and predicted functions of colonic bacterial communities in BALB/c mice, Front. Microbiol., 2017, 165, 13321345.

8 M. Kraler, K. Schedle, K. J. Domig, D. Heine, H. Michlmayr and W. Kneifel, Effects of fermented and extruded wheat bran on total tract apparent digestibility of nutrients, minerals and energy in growing pigs, Anim. Feed Sci. Technol., 2014, 197, 121-129.

9 J. Y. Lim, G. M. Jang, C. V. Garcia and S. P. Lee, Fortification of bioactive compounds in roasted wheat bran by solid-state fermentation using bacillus subtilis HA, Food Sci. Technol. Res., 2017, 23, 395-402.

10 Y. Vazana, Y. Barak, T. Unger, Y. Peleg, M. Shamshoum, T. Ben-Yehezkel, Y. Mazor, E. Shapiro, R. Lamed and E. A. Bayer, A synthetic biology approach for evaluating the functional contribution of designer cellulosome components to deconstruction of cellulosic substrates, Biotechnol. Biofuels, 2013, 6, 182.

11 S. Miyauchi, V. S. Te'o, K. M. Nevalainen and P. L. Bergquist, Simultaneous expression of the bacterial Dictyoglomus thermophilum xynB gene under three different Trichoderma reesei promoters, New Biotechnol., 2014, 31, 98-103.

12 E. V. Stepanova, O. V. Koroleva, L. G. Vasil'Chenko, K. N. Karapetian and M. L. Rabinovich, Fungal decomposition of oat straw during liquid and solid-state fermentation, Prikl. Biokhim. Mikrobiol., 2003, 39, 65-74.

13 K. Schedle, M. W. Pfaffl, C. Plitzner, H. H. Meyer and W. Windisch, Effect of insoluble fibre on intestinal morphology and mRNA expression pattern of inflammatory, cell cycle and growth marker genes in a piglet model, Arch. Anim. Nutr., 2008, 62, 427-438.

14 M. Kraler, K. Schedle, C. Schwarz, K. J. Domig, M. Pichier and A. Oppeneder, Fermented and extruded wheat bran in piglet diets: impact on performance, intestinal morphology, microbial metabolites in chyme and blood lipid radicals, Arch. Anim. Nutr., 2015, 69, 378-398.

15 National Research Council, Nutrient requirements of swine, National Academies Press, Washington, DC, 11th edn, 2012.

16 P. Van Leeuwen, A. Velman, S. Boisen, K. Deuring, G. J. Van Kempen, G. B. Derksen, M. W. Verstegen and G. Schaafsma, Apparent ileal dry matter and crude protein digestibility of rations fed to pigs and determined with the use of chromic oxide $\left(\mathrm{Cr}_{2} \mathrm{O}_{3}\right)$ and acid-insoluble ash as digestive markers, Br. J. Nutr., 1996, 76, 551.

17 Y. Liu, M. Song, T. M. Che, J. A. S. Almeida, J. J. Lee, D. Bravo, C. W. Maddox and J. E. Pettigrew, Dietary plant extracts alleviate diarrhea and alter immune responses of weaned pigs experimentally infected with a pathogenic Escherichia coli, J. Anim. Sci., 2013, 91, 5294-5306.

18 G. Reid, M. B. Kirschner and V. N. Zandwijk, Circulating microRNAs: Association with disease and potential use as biomarkers, Crit. Rev. Oncol. Hematol., 2010, 80, 193-208.

19 M. Deepak and B. Sheweta, Composition, properties and health benefits of indigestible carbohydrate polymers as dietary fiber: a review, Int. J. Biol. Macromol., 2013, 61, 1-6.

20 F. Molist, M. Van Oostrum, F. J. Perez, G. G. Mateos, C. M. Nyachoti and P. J. Van Der Aar, Relevance of functional properties of dietary fiber in diets for weanling pigs, Anim. Feed Sci. Technol., 2014, 189, 1-10.

21 D. Mudgil and S. Barak, Composition, properties and health benefits of indigestible carbohydrate polymers as dietary fiber: a review, Int. J. Biol. Macromol., 2013, 61, 1-6.

22 D. J. Rose, J. A. Patterson and B. R. Hamaker, Structural differences among alkali soluble arabinoxylans from maize (Zea mays), rice (Oryza sativa), and wheat (Triticum aestivum) brans influence human fecal fermentation profiles, J. Agric. Food Chem., 2010, 58, 493-499.

23 B. A. Williams, D. Mikkelsen, L. L. Paih and M. J. Gidley, In vitro fermentation kinetics and end-products of cereal arabinoxylans and $(1,3 ; 1,4)$-beta-glucans by porcine faeces, J. Cereal Sci., 2011, 53, 53-58.

24 L. A. F. Pascoal, M. C. Thcomaz, P. H. Watanabe, U. D. S. Ruiz, A. B. Amorim, E. Daniel and S. Z. D. Silva, Purified cellulose, soybean hulls and citrus pulp as a source of fiber for weaned piglets, Sci. Agric., 2015, 72, 400410. 
25 Y. T. Chu, C. T. Lo, S. C. Chang and T. T. Lee, Effects of Trichoderma fermented wheat bran on growth performance, intestinal morphology and histological findings in broiler chickens, Ital. J. Anim. Sci., 2016, 16, 82-92.

26 M. S. Hedemann, M. Eskildsen, H. N. Laerke, C. Pedersen, J. E. Lindberg, P. Laurinen and K. E. B. Knudsen, Intestinal morphology and enzymatic activity in newly weaned pigs fed contrasting fiber concentrations and fiber properties, J. Anim. Sci., 2006, 84, 1375-1386.

27 H. Chen, X. B. Mao, J. He, B. Yu, Z. Q. Huang, J. Yu, P. Zheng and D. W. Chen, Dietary fibre affects intestinal mucosal barrier function and regulates intestinal bacteria in weaning piglets, Br. J. Nutr., 2013, 110, 1837-1848.

28 Y. Li, W. Jogunoori, W. G. Yao, H. Cao, B. Mishra and L. Mishra, Abstract 4258: Role of $\beta 2 S P$ in asymmetric division and differentiation of intestine epithelial cells, Cancer Res., 2010, 70, 4258-4258.

29 M. Zhang, Y. L. Shan, H. T. Gao, B. Wang, X. Liu, Y. Y. Dong, X. M. Liu, N. Yao, Y. G. Zhou, X. W. Li and H. Y. Li, Expression of a recombinant hybrid antimicrobial peptide magainin II-cecropin $B$ in the mycelium of the medicinal fungus Cordyceps militaris and its validation in mice, Microb. Cell Fact., 2018, 17, 18.

30 H. Ito, M. Satsukawa, E. Arai, K. Sugiyama, K. Sonoyama, S. Kiriyama and T. Morita, Soluble fiber viscosity affects both goblet cell number and small intestine mucin secretion in rats, J. Nutr., 2009, 139, 1640-1647.

31 K. A. Lien, W. C. Sauer and J. M. He, Dietary influences on the secretion into and degradation of mucin in the digestive tract of mono-gastric animals and humans, J. Anim. Feed Sci., 2001, 10, 223-245.

32 H. C. Clevers and C. L. Bevins, Paneth Cells: Maestros of the Small Intestinal Crypts, Annu. Rev. Physiol., 2013, 75, 289-311.

33 J. F. Daudelin, M. Lessard, F. Beaudoin, E. Nadeau, N. Bissonnette, Y. Boutin, J. P. Brousseau, K. Lauzon and J. M. Fairbrother, Administration of probiotics influences F4 (K88)-positive enterotoxigenic Escherichia coli attachment and intestinal cytokine expression in weaned pigs, Vet. Res., 2011, 42, 69.

34 X. Zeng, L. T. Sunkara, W. Jiang, M. Bible, S. Carter, X. Ma, S. Qiao and G. Zhang, Induction of porcine host defense peptide gene expression by short-chain fatty acids and their analogs, PLoS One, 2013, 8, e72922.

35 H. T. Xiong, B. X. Guo, Z. S. Gan, D. G. Song, Z. Q. Lu, H. B. Yi, Y. M. Wu, Y. Z. Wang and H. H. Du, Butyrate upregulates endogenous host defense peptides to enhance disease resistance in piglets via histone deacetylase inhibition, Sci. Rep., 2016, 6, 27070.

36 J. B. Zhao, L. Ping, Y. Wu, P. Guo, L. Liu, N. Ma, L. Crystal, Y. Chen, J. Zhao and J. Zhang, Dietary Fiber Increases Butyrate-Producing Bacteria and Improves the Growth Performance of Weaned Piglets, J. Agric. Food Chem., 2018, 6, 1-30.

37 S. C. Talker, T. Käsera, K. Reutner, C. Sedlka, K. H. Mair, H. Koinig, R. Graage, M. Viehmann, E. Klingler, A. Ladinig, M. Ritzman, A. Saalmmüller and W. Gerner, Phenotypic maturation of porcine NK- and T-cell subsets, Dev. Comp. Immunol., 2013, 40, 51-68.

38 K. E. Keppel, K. L. Campbell, F. A. Zuckermann, E. A. Greeley, D. J. Schaeffer and R. J. Husmann, Quantitation of canine regulatory $\mathrm{T}$ cell populations, serum interleukin-10 and allergen-specific IgE concentrations in healthy control dogs and canine atopic dermatitis patients receiving allergen-specific immunotherapy, Vet. Immunol. Immunopathol., 2008, 123, 337-344.

39 Q. Qian, P. Li, T. C. Wang, J. Zhang, S. F. Yu, T. Chen, L. Yan, Y. S. Song, X. H. Liu, Y. G. Gu, Y. Wang and G. Jia, Alteration of Th1/Th2/Th17 cytokine profile and humoral immune responses associated with chromate exposure, Occup. Environ. Med., 2013, 70, 697-702.

40 H. Antosz, K. Wojciechowska, J. Sajewicz, D. Choroszyńska, B. Marzec-Kotarska, M. Osiak, N. Pajak, W. Tomczak, M. J. Baszak and J. Baszak, IL-6, IL-10, c-Jun and STAT3 expression in B-CLL, Blood Cells, Mol., Dis., 2015, 54, 258265.

41 C. Wang, H. Sun, Y. Song, Z. Ma, G. Zhang, X. Gu and L. Zhao, Pterostilbene attenuates inflammation in rat heart subjected to ischemia-reperfusion: role of TLR4/NF-кB signaling pathway, Int. J. Clin. Exp. Med., 2015, 8, 14565.

42 A. J. Leonel and J. IAlvarezleite, Butyrate: implications for intestinal function, Curr. Opin. Clin. Nutr. Metab. Care, 2012, 15, 474-479. 